\title{
A Radio Survey of Older T Tauri Stars in the $\eta$ Chamaeleontis Cluster
}

\author{
Eric E. Mamajek, ${ }^{1,2}$ Warrick A. Lawson ${ }^{1}$ and Eric D. Feigelson ${ }^{3}$ \\ ${ }^{1}$ School of Physics, University College UNSW, Australian Defence Force Academy, \\ Canberra, ACT 2600, Australia \\ eem@ph.adfa.edu.au, wal@ph.adfa.edu.au \\ ${ }^{2}$ J. William Fulbright Fellow. Present address: Department of Astronomy, \\ University of Arizona, Tucson, AZ 85721, USA \\ ${ }^{3}$ Department of Astronomy \& Astrophysics, Pennsylvania State University, \\ University Park, PA 16802, USA \\ edf@astro.psu.edu
}

Received 1999 June 24, accepted 1999 August 19

\begin{abstract}
A radio continuum survey of X-ray-identified weak-lined $\mathrm{T}$ Tauri stars (WTTs) in the newly-discovered $\eta$ Chamaeleontis cluster has been completed using the Australia Telescope Compact Array (ATCA). The 10 known WTTs in the cluster form a unique sample of codistant late-type pre-main-sequence stars with ages of $\sim 8$ Myr and masses ranging from $0 \cdot 1-1 \cdot 0 \mathrm{M}_{\odot}$. Our survey detected none of the 10 X-ray-emitting WTTs with $3 \sigma$ sensitivity limits at 4.8 and $8.6 \mathrm{GHz}(6 \cdot 2$ and $3.5 \mathrm{~cm})$ of typically $0.4 \mathrm{mJy}$, corresponding to a radio luminosity of $4.5 \times 10^{15} \mathrm{erg} \mathrm{Hz}^{-1} \mathrm{~s}^{-1}$. Rotation periods for these stars indicate that they are not, as a group, fast-rotating stars. The non-detection in the radio bands supports the findings of other radio surveys of inhomogeneous samples of young stars, where radio emission is fairly common (10-30\%) among very young $\mathrm{T}$ Tauri stars across all late spectral types, but confined to rapidly-rotating F-G-K stars amongst older zero-age main sequence stars. Rotation, more than youth, appears to be the key to radio emission in young stars.
\end{abstract}

Keywords: open clusters and associations: $\eta$ Chamaeleontis - stars: pre-main-sequence — radio continuum: stars

\section{Introduction}

Multiwavelength observations over the last two decades have shown that magnetic activity in young pre-main-sequence (pre-MS) stars or T Tauri stars (TTs) is greatly enhanced compared to the mainsequence Sun. The enhanced solar-type activity in TTs likely results from the interaction between the young stars' rapid rotation and their deep convective envelopes through the dynamo mechanism. The enhanced stellar dynamo in T Tauri stars manifests itself through various diagnostics: active chromospheric emission ( $\mathrm{CaII} \mathrm{H}$ and $\mathrm{K}, \mathrm{H} \alpha$, etc.), coronal thermal X-ray emission, and strong nonthermal radio emission (see reviews by Feigelson \& Montmerle 1999; Montmerle et al. 1993; Bertout 1989). The majority of a star's pre-MS lifetime is spent as a weak-lined T Tauri (WTT) star, where the star-disk interaction has ceased, and the subsequent angular momentum evolution of the young star is no longer affected by the circumstellar disk.

In active late-type stars, microwave radio emission $\left(L_{R}\right)$ is proportional to the soft X-ray emission $\left(L_{X}\right)$ from high-energy stellar activity over 10 orders of magnitude in $L_{R}$, ranging from solar microflares to RS CVn binaries (Benz \& Güdel 1994). This remarkable power-law relation appears to become microwave-luminous for the very strongest stellar $\mathrm{X}$-ray sources, an effect tied to the higher coronal temperatures among rapidly-rotating stars with convective envelopes. Stellar X-ray spectra are characterised by soft $(\sim 1-2 \mathrm{MK})$ 'coronal' components and hard $(\sim 5-100 \mathrm{MK})$ 'flare' components. The 'flare' components decay with rotation and age such that their contribution is negligible for most stars older than 1 Gyr (Güdel, Guinan \& Skinner 1997). Highly variable, circularly polarised gyrosynchotron radio emission is associated with hard X-ray emission in flares. The existence of magnetic fields up to thousands of Gauss in strength covering much of the surface of TTs is supported by Doppler imaging and Zeeman measurements (Feigelson \& Montmerle 1999).

The emerging picture is that the vigorous magnetic dynamos of rapidly rotating young solar-type stars produce surface fields which in turn undergo violent reconnection to X-ray-bright plasmas and nonthermal electron populations that produce gyrosynchotron 
emission. Although stellar age was classically the principal variable upon which activity indicators hinged (Skumanich 1972), evidence has accumulated that rotation, not age, is the key to determining a star's activity (see the review by Simon 1992). Radio band studies of active stars with different ages and rotation rates can test this idea.

To date, radio surveys have been conducted for very young $\mathrm{T}$ Tauri stars (both classical and weaklined TTs with ages of $\leq 2 \mathrm{Myr}$ ) in the nearby Taurus (O'Neal et al. 1990; Chiang et al. 1996), $\rho$ Ophiuchi (Leous et al. 1991), Chamaeleon and Corona Australis (Brown et al. 1996) and $\mathrm{LkH} \alpha$ 101 cluster (Stine \& O’Neal 1998) star-forming regions. The two major studies of young WTTs in Taurus-Auriga found radio detection rates of $\sim 10-25 \%$. O'Neal et al. (1990) found $8 \%$ of 119 WTT stars had $6 \mathrm{~cm}(4.8 \mathrm{GHz})$ radio fluxes at the $\log \left(L_{R}\right)>16 \cdot 2 \mathrm{erg} \mathrm{Hz}^{-1} \mathrm{~s}^{-1}$ level. Chiang et al. (1996) found $7 / 28(25 \%)$ of low-mass WTTs (K7-M4) were radio-emitting at $3.6 \mathrm{~cm}(8 \mathrm{GHz})$ in the range $15 \cdot 4<\log \left(L_{R}\right)<16.7 \mathrm{erg} \mathrm{Hz}^{-1} \mathrm{~s}^{-1}$. The detected objects in the O'Neal sample ranged in spectral type from $\mathrm{G} 2-\mathrm{M} 0 \cdot 5$, and most had $\log \left(\mathrm{L}_{X}\right)$ $>30 \cdot 0 \mathrm{erg} \mathrm{s}^{-1}$.

Both homogeneous and inhomogeneous samples of older TTs have also been surveyed for radio emission. The WTTs in the Upper Sco subgroup of the Sco Cen OB Association (Sco OB2) are the only radio-surveyed sample of older T Tauri stars (5 Myr; de Geus, de Zeeuw \& Lub 1989). The Upper Sco WTTs were surveyed in radio by Brown et al. (1996), who examined 20 stars from the X-ray list of Walter et al. (1994). They detected 10/20 WTTs ranging in spectral type from $\mathrm{G} 0-\mathrm{M} 2 \cdot 5$, and with a wide range of rotational velocities $\left(15<v \sin i<77 \mathrm{~km} \mathrm{~s}^{-1}\right)$. It should be noted that $4 / 8$ of radio-detected, Upper Sco WTTs with known $v \sin i$ measurements had $v \sin i>30 \mathrm{~km} \mathrm{~s}^{-1}$, and all 10 of the detections had $\log \left(L_{X}\right) \geq 30 \cdot 0 \mathrm{erg} \mathrm{s}^{-1}$.

Widely dispersed X-ray-discovered active Li-rich stars found by the ROSAT All Sky Survey (RASS) contain both very young WTT stars dispersed from their star-forming regions, and older, low-mass ZAMS (zero-age main sequence) stars (e.g. Covino et al. 1997). The largest samples of RASS stars examined in the radio are the surveys near the Taurus region by Carkner et al. (1997; C97) and Magazzú et al. (1999). The radio detections of C97 had radio luminosity densities $\log \left(L_{R}\right)=15 \cdot 5-$ $16 \cdot 7 \mathrm{erg} \mathrm{Hz}^{-1} \mathrm{~s}^{-1}$. These stars have spectral types G1-M3 $\cdot 5$, and strong X-ray emission with $\log \left(L_{X}\right)>$ $29 \cdot 7 \mathrm{erg} \mathrm{s}^{-1}$. Among the definite $(>4 \sigma)$ detections in C97's sample, whose rotational periods and velocities were determined photometrically by Bouvier et al. (1997), the radio-emitters were predominantly fast-rotating $\mathrm{G}$ and $\mathrm{K}$ stars $\left(\left\langle v_{\text {eq }}\right\rangle=55 \mathrm{~km} \mathrm{~s}^{-1}\right)$. Another radio survey of an inhomogeneous sample of 32 X-ray-selected young stars by White, Pallavicini \& Kundu (1992) found many 8-20 Myr stars with $\log \left(L_{R}\right)=15 \cdot 8-16 \cdot 2 \mathrm{erg} \mathrm{Hz}^{-1} \mathrm{~s}^{-1}$, whereas most of the older ZAMS-age stars were not detected. A number of nearby young, fast-rotating G-K ZAMS \& WTT dwarfs like AB Dor, PZ Tel, and 'Speedy Mic' (HD 197890) have also been found to be variable radio-emitters. These stars have a quiescent state at the $1-3 \times 10^{15} \mathrm{erg} \mathrm{Hz}^{-1} \mathrm{~s}^{-1}$ level, and flare at levels 10-50 times higher (Lim et al. 1992). Among older stars, coeval populations of 100-Myr-old ZAMS stars like the Pleiades have been observed with little success (Bastian, Dulk \& Slee 1988) except for weak radio detections of a few 'ultra-fast rotators' (UFRs; Lim \& White 1995).

The theme emerging from these radio surveys of young stars is that higher nonthermal radio emission exists amongst younger X-ray-luminous populations, and that typically only the fastest rotators amongst ZAMS groups are detectable radio-emitters.

We report here a survey of a unique group of WTTs with ages between those of the youngest $\mathrm{T}$ associations (e.g. Taurus) and older ZAMS clusters like the Pleiades, to explore the association between rotation, youth, and radio emission in that age gap. Our target stars are the X-ray-luminous WTT members of the recently-discovered $\eta$ Chamaeleontis cluster (Mamajek, Lawson \& Feigelson 1999; hereafter MLF99). The cluster's Hertzsprung-Russell diagram shows that these stars have ages of $8 \pm 4 \mathrm{Myr}$, and there is strong evidence that they were born from the same cloud and are codistant at $d=97 \mathrm{pc}$. This sample alleviates the uncertainties of estimating distances and ages that beset most studies of RASS young stars.

To predict the levels of radio emission expected from this older $\mathrm{T}$ Tauri population, we examined the $L_{X}$ and $L_{R}$ relations for active stars. Among the more active stars, $\log \left(L_{R}\right)=\log \left(L_{X}\right)-\log (\kappa)-$ $15 \cdot 5 \pm 0 \cdot 5$, where $L_{X}$ is measured in erg s${ }^{-1}, L_{R}$ is in erg $\mathrm{Hz}^{-1} \mathrm{~s}^{-1}$, and $\kappa=0 \cdot 17$ for RS CVn binaries, Algols, FK Comae stars, and WTTs (Benz \& Güdel 1994). This relation should apply to the $\eta$ Cha WTTs as their $L_{X} / L_{b o l}$ ratios are near the activity saturation level of $10^{-3}$ (MLF99). The $L_{X} / L_{R}$ correlation has little dispersion among simultaneous and near-simultaneous observations [e.g. Güdel et al. (1993) for dMe stars; Fox et al. (1994) for RS CVn binaries], but a $\pm 0 \cdot 5-1$ dex spread is present in non-simultaneous data due to flares and rotational modulation of emitting structures (e.g. Carkner et al. 1997 for WTT stars). This dispersion can be expected for the $\eta$ Cha cluster WTTs, as eight stars exhibited X-ray variability ranging from factors of

\footnotetext{
${ }^{1}$ Note that we include microwave surveys at both 8 and $4.8 \mathrm{GHz}$ in our discussion since the emission from young stars is usually spectrally flat (Güdel \& Benz 1993).
} 
2-7 over $\sim 20 \%$ of the HRI observations (Mamajek, Lawson \& Feigelson 2000). From this analysis, we predict that a few of our brightest X-ray-emitting WTTs should be detectable radio-emitters at the $\log \left(L_{R}\right)=16 \mathrm{erg} \mathrm{Hz}^{-1} \mathrm{~s}^{-1}$ level at any given time.

\section{Observations and Results}

We observed the $10 \eta$ Cha T Tauri stars listed in MLF99 at $4 \cdot 8 \mathrm{GHz}$ and $8 \cdot 6 \mathrm{GHz}(6 \cdot 2$ and $3 \cdot 5 \mathrm{~cm})$ with the Australia Telescope Compact Array (Paul Wild Observatory) in $6 \mathrm{~km} \mathrm{C}$ mode. The phase centres were positioned $10^{\prime \prime}$ north of the stars' optical positions to avoid any possible effects of DC offsets. With $20 \cdot 5$ hours of observing time allocated on 1999 Mar 7-8, each object was observed nine times, with most of the observations taken in 10 minute blocks. Shorter blocks ( 8 minutes) were used near the end of the run to accommodate a ninth observation for all of the objects. The final 12 hours of observation were impaired due to the failure of antennae 6 , hence roughly half of the observations employ only five antenna and have only 10 of the normal number of baselines (15). The antenna failure resulted in theoretical $\mathrm{rms}$ noise values increasing from $0 \cdot 074$ mJy to $0 \cdot 11 \mathrm{mJy}$. The ATCA primary calibrator PKS B1934-638 was observed at the beginning of the run for $\sim 5$ minutes. B1057-797 was used as a secondary phase calibrator, and was observed after every $2 \times 10$-minute block of on-source time. The weather was fair throughout the observations.

Data analysis of the radio fields was performed using the MIRIAD 1.0 package. ${ }^{2}$ Unusually high correlations (presumably due to radio interference) were clipped when examining the UV data, and by flagging extraneous correlations in the real-versusimaginary amplitude diagram. The overall data quality was excellent, and among all of the 4.8 and $8.6 \mathrm{GHz} \mathrm{UV}$ data sets, less than $0.6 \%$ of the correlations were clipped from each set. A 'dirty' image of each field and frequency UV data set was made using the MIRIAD task INVERT in natural weighting mode. The average theoretical rms noise $(\sigma)$ for the $\eta$ Cha cluster member maps was $0 \cdot 11$ mJy beam ${ }^{-1}$, and the measured background noise values for 14 of the 20 dirty images was just slightly higher than the theoretical values (usually around $0 \cdot 13 \mathrm{mJy}$ beam $^{-1}$ ). From these 14 featureless dirty maps (both frequencies for RECX 1, 7, 11, 12 and the $8.6 \mathrm{GHz}$ maps for RECX 3, 4, 5, 6, 9 and 10), a search for detections was conducted immediately and no further processing was warranted. The other six dirty images had higher noise values $(0 \cdot 15<$ $\sigma<0.5$ mJy beam $^{-1}$ ) and required CLEANing to remove the effects of bright confusing sources within or near the primary beam.

Final maps were corrected for primary beam sensitivity. The radio position of the secondary calibrator B1057-797 was precisely as listed in the AT catalog of calibrator sources, indicating that no significant deviations in the optical-radio reference frame should exist in the synthesised maps. A $~ 1$ $\operatorname{arcmin}^{2}$ square box map of each source at each frequency was made and examined in a preliminary search for detections. MIRIAD's statistical programs IMSTAT and HISTO were used to find the position and peak of the flux in a $20^{\prime \prime} \times 20^{\prime \prime}$ box coincident with the optical position.

No $>3 \sigma$ peaks were found within $2^{\prime \prime}$ of the optical positions. Only one target, RECX 10, had a radio source within 1 arcmin of the X-ray star: a $4.5 \sigma$ source with $1.1 \mathrm{mJy}(0 \cdot 7 \mathrm{mJy})$ at $4.8 \mathrm{GHz}(8 \cdot 6 \mathrm{GHz})$ was found $13 \cdot 1^{\prime \prime} \mathrm{NW}$ of RECX 10 at 8:44:30·65, $-78: 46: 19 \cdot 5$ (J2000). The source has a double morphology with component separation of $4^{\prime \prime}$, and is almost certainly an unrelated background double radio galaxy. ${ }^{3}$ To investigate the

Table 1. Radio observations of $\eta$ Cha Cluster T Tauri stars

\begin{tabular}{|c|c|c|c|c|c|c|c|c|}
\hline \multirow{3}{*}{$\begin{array}{l}\text { Star } \\
\text { RECX } \\
\#\end{array}$} & \multicolumn{2}{|c|}{ Optical positions } & \multirow{3}{*}{$\begin{array}{l}\text { Spectral } \\
\text { type }\end{array}$} & \multirow{3}{*}{$\begin{array}{l}\log \left(L_{X}\right) \\
\left(\operatorname{erg~s}^{-1}\right)\end{array}$} & \multirow{3}{*}{$\begin{array}{c}4 \cdot 8 \mathrm{GHz} \\
\text { flux } \\
(\mathrm{mJy})\end{array}$} & \multirow{3}{*}{$\begin{array}{c}4 \cdot 8 \mathrm{GHz} \\
\log \left(L_{R}\right) \\
\left(\operatorname{erg~Hz}{ }^{-1} \mathrm{~s}^{-1}\right)\end{array}$} & \multirow{3}{*}{$\begin{array}{c}8 \cdot 6 \mathrm{GHz} \\
\text { flux } \\
(\mathrm{mJy})\end{array}$} & \multirow{3}{*}{$\begin{array}{c}8 \cdot 6 \mathrm{GHz} \\
\log \left(L_{R}\right) \\
\left(\operatorname{erg~Hz}{ }^{-1} \mathrm{~s}^{-1}\right)\end{array}$} \\
\hline & $\alpha_{J 2000}$ & $\delta_{J 2000}$ & & & & & & \\
\hline & $\mathrm{h} \mathrm{m} \quad \mathrm{s}$ & & & & & & & \\
\hline 1 & $083656 \cdot 2$ & $-785645 \cdot 7$ & $\mathrm{~K} 4$ & $30 \cdot 6$ & $<0 \cdot 34$ & $<15 \cdot 59$ & $<0 \cdot 37$ & $<15 \cdot 62$ \\
\hline 3 & $084137 \cdot 2$ & $-790330 \cdot 9$ & M3 & $29 \cdot 1$ & $<0 \cdot 46$ & $<15 \cdot 71$ & $<0 \cdot 37$ & $<15 \cdot 62$ \\
\hline 4 & $084223 \cdot 7$ & $-790403 \cdot 6$ & $\mathrm{~K} 7$ & $30 \cdot 1$ & $<0 \cdot 31$ & $<15 \cdot 54$ & $<0 \cdot 34$ & $<15 \cdot 59$ \\
\hline 5 & $084227 \cdot 3$ & $-785748 \cdot 5$ & M5 & $29 \cdot 0$ & $<0 \cdot 42$ & $<15 \cdot 68$ & $<0 \cdot 33$ & $<15 \cdot 57$ \\
\hline 6 & $084239 \cdot 0$ & $-785443 \cdot 5$ & M2 & $29 \cdot 5$ & $<0.52$ & $<15 \cdot 77$ & $<0 \cdot 37$ & $<15 \cdot 62$ \\
\hline 7 & $084307 \cdot 7$ & $-790452 \cdot 3$ & K3 & $30 \cdot 3$ & $<0 \cdot 36$ & $<15 \cdot 61$ & $<0 \cdot 40$ & $<15 \cdot 66$ \\
\hline 9 & $084416 \cdot 6$ & $-785908 \cdot 9$ & M4 & $28 \cdot 4$ & $<0 \cdot 40$ & $<15 \cdot 65$ & $<0 \cdot 36$ & $<15 \cdot 61$ \\
\hline 10 & $084432 \cdot 2$ & $-784631 \cdot 7$ & $\mathrm{~K} 7$ & $29 \cdot 8$ & $<0 \cdot 64$ & $<15 \cdot 86$ & $<0 \cdot 44$ & $<15 \cdot 70$ \\
\hline 11 & $084701 \cdot 8$ & $-785935 \cdot 2$ & $\mathrm{~K} 4$ & $30 \cdot 1$ & $<0 \cdot 45$ & $<15 \cdot 70$ & $<0 \cdot 38$ & $<15 \cdot 63$ \\
\hline 12 & $084756 \cdot 9$ & $-785453 \cdot 9$ & M2 & $30 \cdot 1$ & $<0 \cdot 37$ & $<15 \cdot 62$ & $<0 \cdot 35$ & $<15 \cdot 60$ \\
\hline
\end{tabular}

${ }^{2}$ Documentation on MIRIAD (Multichannel Image Reconstruction Image Analysis and Display) is available at http://www.atnf.csiro.au/computing/software/miriad, and was written by Bob Sault and Neil Killeen of ATNF.

\footnotetext{
${ }^{3}$ About $0 \cdot 1$ extragalactic source with $S>0.4 \mathrm{mJy}$ are expected to appear by chance in the $10 \times 1$ arcmin ${ }^{2}$ regions we examined at $8.4 \mathrm{GHz}$ (Windhorst et al. 1993) while $\sim 10$ are expected at $5 \mathrm{GHz}$ (Bridle 1989).
} 
possibility of discovering an $\eta$ Cha radio star that was not in the X-ray sample, we searched for optical counterparts of six bright sources within the primary beams. None were found within $5^{\prime \prime}$ of the radio sources. Finally, we searched unsuccessfully for radio emission from the three early-type members of the cluster: $\eta$ Cha (B8V), RS Cha (A7+A7IV) and HD $75505(\mathrm{~A} 1 \mathrm{~V})$. Table 1 contains stellar data on the $\eta$ Cha cluster WTTs (from MLF99) combined with the upper limit $4 \cdot 8$ and $8 \cdot 6 \mathrm{GHz}$ radio fluxes.

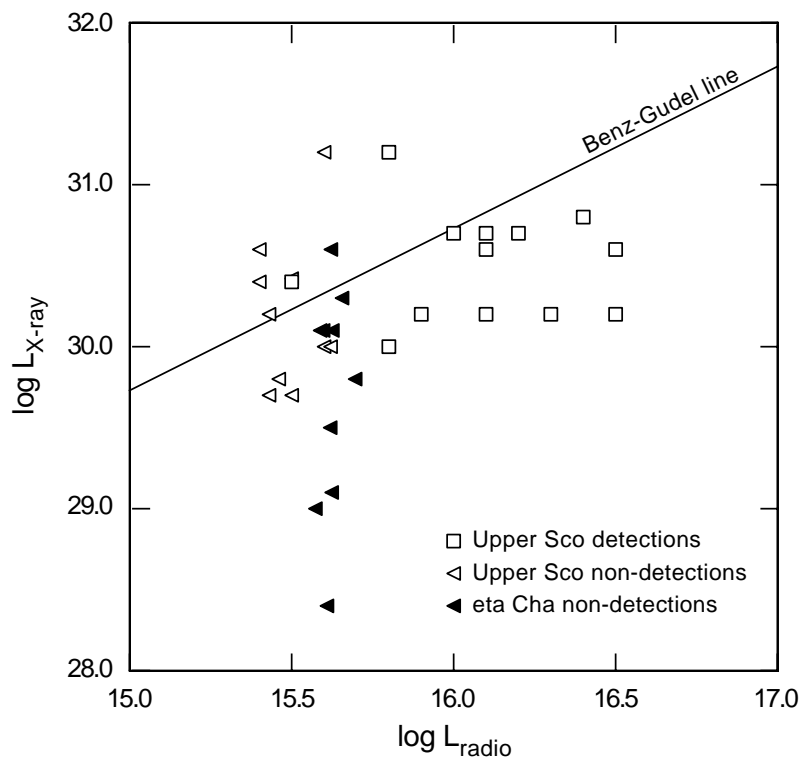

Figure 1-Benz-Güdel diagram for Upper Scorpius and $\eta$ Cha weak-lined $\mathrm{T}$ Tauri stars. The straight line is the expected locus for simultaneous measurements made of active stars. Upper Sco data are from Brown et al. (1996). See text for details.

\section{Discussion}

Figure 1 compares the X-ray and radio levels of the $\sim 8$-Myr-old $\eta$ Cha T Tauri stars and the 5-Myr-old WTTs in the Upper Sco subgroup of the Sco-Cen OB association (Brown et al. 1996) to the Benz \& Güdel (1994) $L_{X} / L_{R}$ relation for stellar activity. Why were none of the $\eta$ Cha WTTs detected with $\log \left(L_{R}\right)>15 \cdot 6 \mathrm{erg} \mathrm{Hz}^{-1} \mathrm{~s}^{-1}$, even when a few quiescent-level radio detections were expected? Flares in stars with lower $L_{X}$ values should also have been seen; past experience has shown that a fraction of younger $\mathrm{T}$ Tauri stars with lower $L_{X}$ values $\left(10^{29}-10^{30} \mathrm{erg} \mathrm{s}^{-1}\right)$ flare during their radio observations (see Figure 5 of C97) at levels an order of magnitude higher than that predicted by the Benz \& Güdel (1994) relation. From an examination of Figure 1, and consideration of the radio detection rates of the surveys mentioned previously, it is surprising that none of our WTTs were detected.

To understand why radio emission was undetected in the $\eta$ Cha WTTs, we look to the stars' rotation as a possible answer. First, we estimated $v \sin i$ from the FWHM of isolated absorption lines in medium- resolution spectra of these stars (as presented in MLF99), comparing them with values of $v \sin i$ measured from high-resolution spectra of RECX 1, 7, 10 and 12 (Covino et al. 1997). Of those four, RECX 7 had the highest $v \sin i\left(28-32 \mathrm{~km} \mathrm{~s}^{-1}\right)$ and the highest FWHM measure among the WTTs in MLF99. Secondly, multi-epoch differential- $V$ CCD photometry of the RECX stars was obtained by W. Lawson and L. Crause during 1999 Feb-Mar using the SAAO $1.0 \mathrm{~m}$ telescope. Preliminary results for these stars indicate rotation periods of $1 \cdot 3-8$ days, typical for young stars (Crause et al. 2000). Making use of the approximate luminosities of MLF99, we calculate equatorial velocities $v_{\text {eq }}<50 \mathrm{~km} \mathrm{~s}^{-1}$ for all of these stars. Only two (RECX 7 and 12) have $v_{\text {eq }}$ values that would place them near the lower limit of $v \sin i$ for ultrafast rotators $(30 \leq v \sin i$ $\leq 150 \mathrm{~km} \mathrm{~s}^{-1}$; see Soderblom et al. 1993).

In conclusion, we find that none of our $\sim 8$-Myr-old WTTs are radio-emitting at the $\log \left(L_{R}\right)>15 \cdot 6 \mathrm{erg}$ $\mathrm{Hz}^{-1} \mathrm{~s}^{-1}$ level. The radio emission is below that seen in younger $(\leq 5$ Myr-old) samples of $\mathrm{T}$ Tauri stars; however most of the stars detected in those studies were rapidly rotating. Data from two studies (MLF99; Crause et al. 2000) indicate that the $\eta$ Cha WTTs are not, as a group, fast rotators. The results of this radio survey support the view that rotation, not youth, is the dominant factor in accounting for nonthermal radio emission in young stars (Simon 1992).

\section{Acknowledgments}

EEM thanks the J. William Fulbright Fellowship program and the Australian-American Educational Foundation (AAEF) in Canberra for support. EDF was funded by NASA contract NAS8-38252. We would also like to thank Dave Rayner, who was duty astronomer during our ATCA observations, Paul O'Neil at ADFA for help with observations, Bob Sault at ATNF/Epping for assistance with data analysis, and Steve James at ADFA for help with data extraction. The Australia Telescope (ATCA) is funded by the Commonwealth of Australia for operation as a National Facility managed by CSIRO. We acknowledge the use of NASA's SkyView facility (http://skyview.gsfc.nasa.gov) located at the NASA Goddard Space Flight Center.

\section{References}

Bastian, T. S., Dulk, G. A., \& Slee, O. B. 1988, AJ, 95, 794 Benz, A. O., \& Güdel, M. 1994, A\&A, 285, 621

Bertout, C. 1989, ARA\&A, 27, 351

Bouvier, J., et al. 1997, A\&A, 318, 495

Bridle, A.H. 1989, Synthesis Observing Strategies - A 'HitchHiker's Guide' in Synthesis Imaging in Radio Astronomy, Lectures from the 3rd NRAO Synthesis Imaging Summer School, vol. 6, ed. R. A. Perley, F. R. Schwab, \& A. H. Bridle (San Francisco: ASP), p. 443 
Brown, A., Walter, F. M., Ambruster, C., Stewart, R. T., \& Jeffries, R. 1996. in Radio Emission from the Stars and the Sun, ASP Conf. Ser. 93, ed. A. R. Taylor \& J. M. Paredes (San Francisco: ASP), p. 294

Carkner, L., Mamajek, E., Feigelson, E., Neuhäuser, R., Wichmann, R., \& Krautter, J. 1997, ApJ, 490, 735

Chiang, E., Phillips, R. B., \& Lonsdale, C. J. 1996, AJ, 111,355

Covino, E., Alcalá, J. M., Allain, S., Bouvier, J., Terranegra, L., \& Krautter, J. 1997, A\&A, 328, 187

Crause, L. A., Lawson, W. A., Mamajek, E. E., \& Feigelson, E. D. 2000, in preparation

de Geus, E. J., de Zeeuw, P. T., \& Lub, J. 1989, A\&A, 216,44

Feigelson, E. D., \& Montmerle, T. 1999, ARA\&A, in press

Fox, D. C., et al. 1994, A\&A, 284, 91

Güdel, M., \& Benz, A. O. 1993, ApJ, 405, L63

Güdel, M., Guinan, E. F., \& Skinner, S. L. 1997, ApJ, 483, 947

Güdel, M., Schmitt, J. H. M. M., Bookbinder, J. A., \& Fleming, T. A. 1993, ApJ, 415, 236

Leous, J. A., Feigelson, E. D., Andre, P., \& Montmerle, T. 1991, ApJ, 379, 683

Lim, J., Nelson, G. J., Castro, C., Kilkenny, D., \& Van Wyk, F. 1992, ApJ, 388, L27

Lim, J., \& White, S. M. 1995, ApJ, 453, 207
Magazzù, A., Umama, G., \& Martín, E. L. 1999, A\&A, 346,878

Mamajek, E. E., Lawson, W. A., \& Feigelson, E. D. 1999, ApJ, 416, L77 (MLF99)

Mamajek, E. E., Lawson, W. A., \& Feigelson, E. D. 2000, in preparation

Montmerle, T., Feigelson, E. D., Bouvier, J., \& Andre, P. 1993. in Protostars \& Planets III, ed. E. H. Levy \& J. I. Lunine (Tucson: Univ. of Arizona Press), pp. 689-717 O'Neal, D., Feigelson, E. D., Mathieu, R. D., \& Myers, P. C. 1990, AJ, 100,1610

Simon, T. 1992. Seventh Cambridge Workshop on Cool Stars, Stellar Systems, and the Sun, ASP Conf. Ser. 26, ed. M. S. Giampapa \& J. A. Bookbinder (San Francisco: ASP), p. 3

Skumanich, A. 1972, ApJ, 171, 565

Soderblom, D. R., et al. 1993, AJ, 106, 1059

Stine, P. C., \& O'Neal, D. 1998, AJ, 116, 890

Walter, F. M., Vrba, F. J., Mathieu, R. D., Brown, A., \& Myers, P. C. 1994, AJ, 107, 692

White, S. M., Pallavicini, R., \& Kundu, M. R. 1992, A\&A, 257,557

Windhorst, R. A., Fomalont, E. B., Partridge, R. B., \& Lowenthal, J. D. 1993, ApJ, 405, 498 